\title{
La institucionalización y profesionalización de la Sociología en el País Vasco: un proceso inacabado
}

\author{
Institutionalization and professionalization of sociology in the \\ Basque Country: an unfinished process
}

Idoia Martín Aranaga • idoia.martin@ehu.eus

UNIVERSIDAD DEL PAÍS VASCO/EUSKAL HERRIKO UNIBERTSITATEA

UPV/EHU

Recibido: 28/03/2016

Aceptado: 11/06/2016

\section{Resumen}

Desde que se inician los estudios de sociología en 1963 en el Instituto de Estudios Económico-Sociales —después Instituto de Estudios Sociales- de la Universidad de Deusto, han pasado más de 50 años, 50 años de historia de la sociología en el País Vasco. Se considera necesario, por tanto, conocer el pasado de la sociología vasca, para así, quizá, ser capaces de comprender el presente. Este artículo tiene un doble objetivo. En primer lugar, identificar los hechos y las personas representativas que han definido y protagonizado, respectivamente, el proceso de institucionalización y profesionalización de la sociología en el País Vasco. En segundo lugar, y no menos importante para nosotros, verificar la hipótesis planteada que consiste en comprobar, en el caso de la sociología vasca, la existencia de tensiones entre diferentes cosmovisiones o concepciones de la sociología que han atravesado todo el recorrido de la sociología vasca. Nos referimos aquí a las tensiones u oposiciones entre la sociología como disciplina científica y la sociología como disciplina aplicada, entre la sociología teórica y la sociología empírica, la sociología académica y la sociología profesional, entre otras. Y que pensamos que pueden haber provocado que el proceso de profesionalización de la sociología en el País Vasco resulte inacabado.

Palabras clave: institucionalización, profesionalización, sociología, País Vasco, proceso inacabado

\section{Abstract}

Since sociology studies begin in 1963 at the Institute of Economic and Social Studies -later Institute of Social Studies- of the University of Deusto, have passed more than 50 years, 50 years of history of sociology in the Basque Country. It is necessary, therefore, to know the past of the Basque sociology, so, perhaps, be able to understand the present. This article has a double objective. First of all, identify the facts and representative persons who have defined and starred, respectively, the process of institutionalization and professionalization of sociology in the Basque Country. Secondly, and no less important to us, verify the hypothesis which consists, in the case of the Basque sociology, check the existence of tensions between different conceptions of sociology that have gone through the entire route of the Basque sociology. We refer here to the tensions or oppositions between sociology as a scientific discipline and sociology as applied discipline, between theoretical sociology and empirical sociology, academic sociology and professional sociology, among others. And we think that may have led to the professionalization of sociology in the Basque Country resulting unfinished.

Key words: institutionalization, professionalization, sociology, Basque Country, unfinished process. 


\section{INTRODUCCIÓN ${ }^{1}$}

Desde que se inician los estudios de sociología en 1963, en el Instituto de Estudios Económico-Sociales de la Universidad de Deusto, han pasado más de 50 años, 50 años de historia de la sociología en el País Vasco. Se considera necesario, por tanto, conocer el pasado de la sociología vasca, para así, quizá, ser capaces de comprender el presente.

Este artículo tiene un doble objetivo. En primer lugar, identificar los hechos y las personas representativas que han definido y protagonizado, respectivamente, el proceso de institucionalización y profesionalización de la sociología en el País Vasco. En segundo lugar, y no menos importante para nosotros, verificar la hipótesis planteada que consiste en comprobar, en el caso de la sociología vasca, la existencia de tensiones entre diferentes cosmovisiones o concepciones de la sociología, que han atravesado todo el recorrido de la sociología vasca. Nos referimos aquí a las tensiones u oposiciones entre la sociología como disciplina científica y la sociología como disciplina aplicada, entre la sociología teórica y la sociología empírica, la sociología académica y la sociología profesional, entre otras. Y que pensamos que pueden haber provocado que el proceso de profesionalización de la sociología en el País Vasco resulte inacabado.

Así, hemos identificado cuatro momentos o fases del proceso de institucionalización y profesionalización de la sociología en el País Vasco. La primera abarca el periodo 19531973, y en ella destacan como hechos relevantes: el Instituto de Estudios Sociales de la Universidad de Deusto y la cátedra de Sociología de la Facultad de Ciencias Políticas, Económicas y Comerciales de la Universidad de Bilbao. La segunda fase, de 1974 a 1980, comprende la creación de la Facultad de Ciencias Políticas y Sociología en la Universidad de Deusto y la fundación de la Asociación Vasca de Sociología, así como la aparición de las primeras experiencias de sociología aplicada. La tercera es la fase de consolidación de la sociología en el País Vasco, años 1980-1990, con la creación de la titulación de sociología en la Facultad de Ciencias de la Información —más tarde Facultad de Ciencias Sociales y de la Información- de la Universidad del País Vasco (UPV/EHU), y el boom de la demanda de estudios sociológicos. Por último, a partir de los años 90 se produce un crecimiento de la sociología, tanto en términos de plazas ofertadas de profesorado en esa disciplina en la universidad pública vasca, como de titulados en sociología. Ello hasta comienzos de la década de los años 2000, en que comienza a advertirse,

\footnotetext{
${ }^{1}$ Este texto se ha elaborado, en gran parte, gracias a las aportaciones, obtenidas en contexto de "entrevistas en profundidad", realizadas entre 2012-2013, de los catedráticos de Sociología -y se citan en el orden en que fueron entrevistados-: Víctor Urrutia (UPV/EHU), Jon Leonardo (U. de Deusto), Ander Gurrutxaga (UPV/EHU), Alfonso Pérez-Agote (UCM) y José Ignacio Ruiz Olabuenaga (U. de Deusto); pero también de Gonzalo Maestro (catedrático de Derecho Constitucional), Manuel González Portilla (catedrático de Historia Contemporánea), por su conocimiento del periodo estudiado, dada su condición de decanos en los primeros tiempos de la hoy Facultad de Ciencias Sociales y de la Comunicación de la UPV/EHU, y de Imanol Zubero (profesor titular del Departamento de Sociología y Trabajo Social de la UPV/EHU), como Presidente de la Asociación Vasca de Sociología y Ciencia Política. A todos ellos quisiera expresar mi agradecimiento por su receptividad, el tiempo dedicado, el conocimiento transmitido y la generosidad mostrada al ofrecerme pistas que han permitido ir completando, aunque sea someramente, esta aproximación al relato de lo que ha supuesto la institucionalización de la sociología en el País Vasco.
} 
según los interlocutores clave entrevistados, una cierta crisis de la sociología en el País Vasco.

\section{LOS ANTECEDENTES}

Como señala Ruiz Olabuenaga (1998), para llevar a cabo una evaluación de nuestro pasado, es preciso recordar los condicionantes históricos que han determinado la joven existencia de la sociología vasca y su no menos endeble existencia corporativa. Para este autor, «no deja de extrañar que, mientras en Europa, la discusión sociológica era tan frecuente desde la mitad del siglo xix tanto en Inglaterra como en Alemania y Francia, entre nosotros no hubiese quien, al menos como copia e imitación, intentase cultivarla. Especialmente en la sociedad vasca que por su proximidad fronteriza, por la brillante tradición de la Sociedad Bascongada de Amigos del País y, sobre todo, por la revolución industrial inusitadamente rápida desde esa misma mitad del siglo, parece que debería haber asistido a una reflexión sociológica intensa de los cambios sociales que en ella estaban ocurriendo» (Ruiz Olabuenaga, 1998: 9).

Ruiz Olabuenaga (1998) señala tres condicionantes fundamentales que han acompañado el gran cambio ocurrido en la sociedad vasca desde mediados del siglo xix y la ausencia de una reflexión sociológica. Al primero de ellos lo denomina como «el del desierto universitario del Norte», un páramo académico desde Oviedo a Zaragoza en el que no existía una sola universidad pública mientras que sí las había en Santiago, Oviedo, Salamanca o La Laguna en las Islas Canarias (Ruiz Olabuenaga, 1998). Los jesuitas establecieron estudios de Filosofía y Letras, junto a los de Derecho en la Universidad de Deusto, pero la falta de estudiantes les obligó a cerrar la Facultad de letras primero y la de historia después, a pesar de treinta años de existencia en 1916. En segundo lugar, menciona el autor, la no menos esterilizante experiencia de cuarenta y cinco años de guerra civil y de control ideológico dictatorial, con repetidos estados de excepción incluidos. Por lo que «difícilmente podía la Sociología existir como reflexión independiente y autónoma en un marco social en el que todo debía ocurrir como el dictador pretendía» (Ibídem, 1998: 10). Finalmente, la ausencia de la Sociología en toda la sociedad española, incluida la Universidad, no hacía sino favorecer este exilio social de la Sociología en el País Vasco.

Por su parte, para Gurrutxaga (1989) las causas del tardío proceso de institucionalización de la sociología en el País Vasco se deberían a «la persistencia de la tradición, el peso de los sectores eclesiásticos, la falta de modernización política, la carencia de espacios públicos democráticos, la tardía institucionalización de la universidad y la casi nula presencia del sector privado en la organización científica» (Gurrutxaga, 1989: 9).

Sin embargo, según Ruiz Olabuenaga (1998), si no existió teorización sociológica en el sentido estricto del término, sí se puede hablar de ciertas tentativas de reflexión sociológica. Se trata de estudios propios de sociografía más que de Sociología propiamente dicha. En- 
tre todos ellos destaca por su sistematización la Corografía de Larramendi. También la polémica entre Unamuno y Trueba en torno al trabajo, sobre «La organización social de Vizcaya en la primera mitad del siglo XIX», enviado por este último a la Exposición Universal de París de 1867 (Ibídem, 1998). Tampoco se deben olvidar los análisis sociográficos que la condición de los inmigrantes y obreros industriales suscitaron en Lavandivar, Posse Villega, Gumersindo Gómez, Iztueta, en los informes para el Instituto de Reformas Sociales de Madrid, y en los de Alzola, Lazurtegui y otros.

Ya en el año 1917, desde la Revista Hermes, Olascoaga reclamaba la necesidad de una sociología vasca, reclamación que volvía a repetirse, esta vez por parte de Barandiaran, desde el seminario de Vitoria con citas a Durkheim y otros autores de las escuelas francesa y alemana (Ruiz Olabuenaga, 1998).

\section{1953-1973: LOS ORÍGENES}

\subsection{El Instituto de Ciencias Sociales de la Universidad de Deusto: el origen de la sociología académica vasca}

Los orígenes de la actual sociología vasca provienen de la iniciativa de la Compañía de Jesús cuando en 1953 decide implantar en la Universidad de Deusto y, con carácter experimental y privado, unos cursos de formación socioeconómica. En 1963, se encarga de impartir estos estudios el Instituto de Estudios Económico-Sociales, adscrito a la Facultad de Derecho (Setién, 1998).

En 1966 el Instituto cambia de nombre - Instituto de Ciencias Sociales-, se independiza y adopta un plan de estudios de 5 años, al final de los cuales se otorgaba el título privado de licenciatura en Sociología por la Universidad de Deusto² (Universidad de Deusto, 1997). Su nacimiento coincide con el comienzo de la inquietud social y la introducción de la sociología científica, con la incorporación al claustro de varios profesores formados en Estados Unidos, Alemania, Francia e Italia (Setién, 1998). Se trata de un instituto confesional católico seguidor de las tradicionales consignas de León XIII (Ruiz Olabuenaga, 1998), con un plantel de profesores dedicados principalmente a la docencia (Iturrate, 1975).

Así, cabe citar como profesores del Instituto de Ciencias Sociales a (Iturrate, 1975): Aranzadi, director y coordinador del Instituto, profesor de Sistemas Sociales y de Nuevas Formas de Empresa; Ruiz Olabuenaga, que estudió en la Universidad Gregoriana de Roma y en la de Fordham (New York-USA), especializándose en Sociología Urbana y en Sociología de las Organizaciones; Baselga, especializado en Sociología del cambio y conflicto social y Sociología de la Religión; Pujana, formado en la Universidad de Munich; Iturrate, 
que cursó en la Universidad Gregoriana de Roma; Cayero, profesor de Sociología Industrial, que estudió en París, con Reynaud; Martínez de la Pera, que cursó estudios en La Sorbona; Martínez Sheifler, especializada en Sociología de la Religión en la Universidad de Fordham; De Pablo, profesor de Estratificación y Clases Sociales, que estudió en California; Urquijo, profesora de Sociología de la Delincuencia; Uriarte, especializado en Psicología industrial y ergonomía; y Fernández de Luco, profesor de Psicología Social.

En este periodo, guarda también relación con la sociología la obra de algunos profesores de la Facultad de Derecho de la Universidad de Deusto. Es el caso de Verdú, especialista en Ciencia Política y Derecho Constitucional, y Sánchez de la Torre, cuya obra está relacionada con la sociología jurídica.

Para Iturrate (1975), dos líneas generales, entrevistas en algunos autores considerados como promotores de la sociología, subyacen y predominan en el quehacer sociológico de estos años: una empírica y otra crítica. Así, este autor señala:

«La línea empírica trata en sus investigaciones de recoger datos que cubran vacíos informativos y que permitan describir la situación social, detectar sus problemas y factores, y perfilar sus tendencias [...]. Aunque encuadra sus estudios en un marco teórico, tiende a marginar este nivel en el que a grandes rasgos es deudora de la orientación funcionalista. Sus pautas, tomadas de los Estados Unidos, país en que la sociología ha logrado su más alto grado de institucionalización, predominan en nuestro ámbito académico.

La línea crítica se vigoriza en los últimos años con las tensiones experimentadas en el proceso de desarrollo español y en el plano internacional. Su tarea se mueve en un plano teórico interpretativo más acusado [...]. Su fuente de inspiración es el pensamiento científico social principalmente europeo, opuesto al idealismo, empiricismo y funcionalismo. Algunos de sus miembros recuperan orientaciones teóricas y metodológicas de Marx articuladas en diferentes interpretaciones.

Además de su cometido científico una y otra línea presentan potenciales ideológicos diversos [...].

Ambas manifiestan que nuestra sociología depende de la realizada en otros países e importada por los estudiosos que se desplazan a ellos para efectuar o ultimar su especialización. Entre estos países destacan Estados Unidos, Francia, Alemania, Inglaterra e Italia» (Iturrate, 1975: 591-592).

Con esta aportación Iturrate (1975) hace aparecer, en nuestro contexto, las tensiones entre la sociología empírica y la sociología teórica, la sociología norteamericana y la sociología europea, añadiendo además el componente ideológico que acompaña a cada una de ellas. También resalta la dependencia de nuestra sociología de la realizada en otros países, siendo en definitiva importada por los estudiosos que se desplazan a ellos para realizar sus especializaciones. 


\subsection{El origen de la Sociología en la universidad pública del País Vasco: la cátedra de Sociología en la Facultad de Ciencias Políticas, Económicas y Comerciales de Bilbao}

En esta primera fase de institucionalización, «el desarrollo oficial de la sociología en la Universidad [pública] va muy unido al de las Facultades de Ciencias Políticas y Económicas en las que se incluye como disciplina» (Iturrate, 1975: 592). En este sentido, conviene mencionar aquí la cátedra de Sociología ${ }^{3}$, que obtiene Jiménez Blanco (Gurrutxaga, 2013) en 1962, en la Facultad de Ciencias Políticas, Económicas y Comerciales de Bilbao (Beltrán, 2009). Constituye una de las tres primeras cátedras de Sociología obtenidas después de la guerra civil en Facultades de Ciencias Políticas, Económicas y Comerciales en universidades en España, tras la de Gómez Arboleya en 1954 en la Universidad de Madrid y la de Salustiano del Campo en la Universidad de Barcelona también en 1962 (Del Campo, 2001). Y sitúa el origen de la sociología en la universidad pública del País Vasco en los primeros años de la década de 1960 y en la Facultad de Bilbao. Esta facultad, creada a partir de la histórica Escuela de Altos Estudios Mercantiles, y dependiente de la Universidad de Valladolid por estas fechas (De Pablo y Rubio, 2006), sentará las bases de la futura Facultad de Ciencias Económicas y Empresariales de la Universidad de Bilbao ${ }^{4}$, esta última fundada en 1968. De hecho, el establecimiento de esta facultad se considera «la punta de lanza para la petición de una universidad que, dado el ambiente político de la época, no sería una universidad vasca, ni siquiera del País Vasco, sino sólo de Bilbao» (Ibídem, 2006: 45).

La Universidad de Bilbao — que se convertirá posteriormente en la Universidad del País Vasco/Euskal Herriko Unibertsitatea-, cuenta por tanto con cátedra y departamento de sociología ${ }^{5}$. Cabe destacar, en la década de 1970, a Moya, catedrático y Jefe del Departamento de Sociología (Iturrate, 1975) de la Facultad de Ciencias Económicas de la Universidad de Bilbao entre 1971 y 1974 (Ramos, 2013), que amplió estudios con Köning en la Universidad de Colonia y está especializado en Teoría Sociológica (Iturrate, 1975). También son miembros del Departamento en estas fechas: De la Iglesia, Arpal, Pérez-Agote y Larrea.

\footnotetext{
3 Según Ruiz Olabuenaga (2013), en España no existían en esa época los estudios de Sociología en la universidad, pero sí había cátedras de Sociología. Se presentaban a las cátedras de Sociología aquellos que se habían licenciado en derecho y ciencias afines, después se habían marchado a Estados Unidos o a Alemania para realizar la tesis doctoral, y luego volvían a España, donde se les reconocía el título de doctor. Es el caso, por ejemplo, de catedráticos de Sociología como Díez Nicolás, Salustiano del Campo, Jiménez Blanco, Moya. De hecho, Ruiz Olabuenaga (1998) llama a esta sociología, "Sociología híbrida», un hibridismo que, a su entender, tuvo «secuelas perniciosas para la programación de los estudios de Sociología en toda España y, por consiguiente, también en la Sociología vasca» (Ruiz Olabuenaga, 1998: 12).

${ }^{4}$ La Universidad de Bilbao se crea por Decreto Ley 5/68 de junio de 1968 en el marco de los «Planes de Desarrollo" que percibían las dificultades de atender las demandas educativas de crecientes poblaciones urbanas, así como la necesidad de una reforma integral del sistema educativo que se intentaría con la Ley General de Educación de 1970 (Universidad del País Vasco, 2013). La Universidad de Bilbao comienza su andadura con la citada Facultad de Económicas y las nuevas de Medicina y Ciencias, sin distrito universitario propio, y con D. Juan Echevarría Gangoiti como primer Rector.

${ }^{5}$ Como señala Urrutia (2013b), habría que distinguir entre lo que es un departamento desde el punto de vista de la denominación formal y lo que es un departamento cuando ya se constituye como entidad institucionalizada. No será hasta la aprobación de la Ley Orgánica 11/1983, de 25 de agosto, de Reforma Universitaria y, sobre todo, del Real Decreto 2360/1984, de 12 de diciembre, sobre departamentos universitarios, que los departamentos adquirirán la estructura administrativa y organizativa, tal y como la conocemos en la actualidad.
} 


\section{LOS AÑOS 1974-1980: EL DESARROLLO DE LA SOCIOLOGÍA ACADÉMICA Y LOS PRIMEROS INTENTOS DE PROFESIONALIZACIÓN}

Se puede decir que la segunda fase de la institucionalización de la sociología vasca comprende la creación de la Facultad de Ciencias Políticas y Sociología en la Universidad de Deusto en 1974 y de la Asociación Vasca de Sociología en 1977.

\subsection{La creación de la Facultad de Ciencias Políticas y Sociología de la Universidad de Deusto}

En la Universidad de Deusto, la creación de la Facultad de Ciencias Políticas y Sociología por la Santa Sede data de 1974 quedando constituida definitivamente en 1976, aunque únicamente se organizan los estudios de la especialidad de Sociología (Setién, 1998). Sin embargo, habrá que esperar hasta 1977, fecha en la que se aprobó un Real Decreto por el que se reconocían oficialmente los estudios de Ciencias Políticas y Sociología, para que Deusto pudiera organizar un examen de Licenciatura para obtener el título oficial (Ibídem, 1998).

En la fundación de esta facultad participan Aranzadi, Uriarte y Ruiz Olabuenaga, profesores del Instituto de Ciencias Sociales, preocupados por la justicia social (Ruiz Olabuenaga, 2013).

En cuanto al profesorado de esta facultad, a la lista de profesores citados por Iturrate (1975) más arriba, habría que añadir los nombres de Belda, profesor de Historia de las Ideas y Formas Políticas, y Solozabal, de Introducción a la Economía, quienes constituyen la primera generación de profesores siendo la mayoría de ellos filósofos, juristas o economistas. Luego hubo una segunda generación, aquellos que habían terminado la carrera de Sociología en la Universidad de Deusto: Llera, Urrutia, Mardones, Calvo, Yabar (Urrutia, 2012). Y serán también profesores: Leonardo, que realiza estudios de sociología en la Universidad de Deusto; Elzo y Velasco, que lo hacen en la Universidad de Lovaina, y Garmendia.

Para Leonardo (2012), la Facultad de Ciencias Políticas y Sociología de la Universidad de Deusto:

«Fue convirtiéndose poco a poco en el lugar de socialización de los dirigentes sindicales y de los partidos políticos en el País Vasco, con unos debates muy vivos sobre todos los problemas sociales, ya que no podemos olvidar que la Iglesia tenía esa situación de excepcionalidad permitida por el propio régimen de Franco, que permitía desarrollar una serie de temáticas que en otros sitios eran prácticamente imposible. [...] Los debates diríamos ideológicos son muy fuertes en ese momento en el mundo de la sociología» 
Este autor advierte en esta facultad las tensiones ya señaladas entre sociología teórica y sociología empírica, las cuales se identifican con una cierta división ideológica. Según sus palabras:

«En nuestra facultad, aunque es en una escala menor, en ese momento aparece ya un pensamiento diríamos más teórico, más crítico por una parte, que está más en la línea de un pensamiento basado en lo que puede ser el marxismo, la teoría crítica, todo ese tipo de cuestiones. Por ejemplo, José María Mardones hizo la tesis aquí sobre la Escuela crítica de Frankfurt, Andoni Cayero viene del mundo del sindicalismo, está Paco Llera, y luego juega un papel importante en el pensamiento político Rafael Belda. [...]. Y luego la otra orientación está más en la línea de aplicabilidad, de los métodos. Por una razón evidente, porque había gente que trabajaba fuera, en las organizaciones, y eso va a dar una visión mucho más pragmática; bueno, el propio padre Uriarte, que trabaja en el mundo de las organizaciones, de las empresas [...], el peso de Ruiz Olabuenaga [...], Félix Calvo, gente que está en el mundo de la investigación empírica, que va a dar más potencia a esta dimensión. Aquí se producen identificaciones como, por ejemplo, si te dedicabas más a la investigación empírica, a la estadística, pues propiamente eso era una identificación del triunfo de una sociología más funcionalista, más de corte americanista y más colaboracionista con el sistema, y mientras si te dedicabas a... Sí, hoy nos reímos pero era así» (Leonardo, 2012).

También Ruiz Olabuenaga (2013) hace referencia al componente ideológico existente en aquellos momentos en la facultad: «Católico-no católico, nacionalista-no nacionalista, izquierda-derecha. Cada uno de estos grupos, todo esto creaba sus capillas, así no podía funcionar la facultad».

De hecho, este último autor define a la sociología del momento como «Sociología dogmática».

«[La Sociología vasca era] una Sociología dogmática, no tanto, aunque también, por encuadrarse en la estructura confesional católica como la de la universidad de Deusto, sino por colisión implacable de dogmas incompatibles que pretendían, cada uno, por su parte, imponer la reflexión sociológica, la verdad de sus revelaciones históricas. No exagero nada si digo que, en una misma aula los profesores teníamos como oyentes, fervientes seminaristas, frailes y monjas, junto con militantes activos del troskismo o del marxismo ortodoxo, exseminaristas trásfugas de sus seminarios hacia la acción social, cripto nacionalistas y etarras, falangistas y policías secretas [...]. En aquel totum revolutum competían por la exclusividad de su corrección política, la escuela social católica de León XIII, el marxismo, tanto heterodoxo como ortodoxo, el Mayo del 68...» (Ruiz Olabuenaga, 1998: 12). 


\subsection{La Asociación Vasca de Sociología (AVS/ESE) y los primeros intentos de profesionalización de la sociología en el País Vasco}

Otro elemento importante de esta segunda fase de institucionalización de la sociología en el País Vasco es la creación de la Asociación Vasca de Sociología/Euskal Soziologia Elkartea (AVS/ESE), que nace en 1977, aunque su registro legal tiene lugar en 1978, coincidiendo con el proceso democrático español, el dinamismo asociativo que experimentaron los múltiples ámbitos de la sociedad y el empuje de las primeras promociones de sociología surgidas de la Universidad de Deusto (Aierdi, 2007). Es la primera Asociación territorial de sociólogos en España que pronto, junto con otras, formará la Federación de Asociaciones de Sociología del Estado Español (FASEE), en consonancia con el desarrollo autonómico que va experimentando el país (Aierdi, 2007; Urrutia, 2012).

Para Aierdi (2007: 228), «su carácter pionero así como las circunstancias sociopolíticas y académicas del País Vasco han configurado un tipo de asociación mestiza a caballo entre el ámbito profesional y el académico». Es lo que indican las palabras de uno de sus fundadores, Urrutia (2012), quien junto a Llera participó activamente en la fundación de la Asociación Vasca de Sociología:

[Es en] los primeros años del arranque de la democracia, cuando las primeras promociones nos planteamos una herramienta que nos haga ser visibles como profesionales de la sociología, y esta herramienta es la Asociación Vasca de Sociología. [...] Y ahí entró en discusión una cuestión importante que es si esas asociaciones, en concreto la Asociación Vasca de Sociología, se podía convertir o no en un colegio profesional. Porque el ser colegio te daba una ventaja económica porque disfrutabas de unos apoyos en la administración, pero también obligaba a una serie de normas y de controles a los cuales aquellos sociólogos rebeldes no estábamos dispuestos. Entendíamos que constituirnos en colegio nos quitaba libertad para expresar nuestras ideas y para mantener una línea de la sociología orientada hacia el cambio social y político. [...]. Y podemos decir que la Asociación es una asociación de carácter muy mestizo, muy mixto entre lo que es una asociación entendida como agrupación de personas que se interesan por la sociología desde el punto de vista científico y, por otra parte, que tenía los objetivos puestos en el desarrollo e implantación de una profesión (Urrutia, 2012).

Así, las promociones de los años 1970, motivadas «a lo largo de su formación universitaria por una cultura del compromiso orientado a las transformación política, mantuvieron durante un tiempo esta pulsión crítica, fundamentalmente de raíz marxista y poco proclive a una integración profesional "sin más»» (Aierdi, 2007: 228). Para Aierdi (2007), ello respondía al componente vocacional de unos estudios que por aquel entonces se consideraban sobre todo una herramienta del conocimiento necesaria para la lucha política.

Poco a poco la tendencia profesional irá imponiéndose, dadas las necesidades marcadas por la creciente demanda profesional, y en la que también intervendrá la "alarmante» tasa de paro de los sociólogos en ese tiempo y la ausencia de una «tipificación profesional» 
(Ibídem, 2007). Además, señala Aierdi (2007: 228), «la percepción social de la Sociología como profesión estaba huérfana de referencias concretas tanto en los medios de comunicación como en la vida cotidiana».

Por otro lado, a finales de la década de 1970, se inician las primeras experiencias de una sociología más profesional. Es el caso de AZTERKA, grupo de investigación social aplicada fundado por Urrutia y Llera en 1978, y del que también son miembros Narvaiza, González, Gurrutxaga y Puente, que inicia «los primeros trabajos de investigación empírica de sociología aplicada, de corte profesional» (Urrutia, 2012, 2013a) Y del gabinete de sociología CINDES (Centro de Investigación de Expectativas Sociales) (Gobierno Vasco, 2013), creado por Ruiz Olabuenaga, también en esos años (Urrutia, 2013a).

\section{1980-1990: LA CONSOLIDACIÓN DE LA SOCIOLOGÍA EN EL PAÍS VASCO}

La «explosión» democrática va a crear un nuevo clima. Según Gurrutxaga (1989: 9), «la transición democrática genera la «necesidad» del sociólogo. Hay que volver a pensar las bases del funcionamiento del orden social y político y, sobre todo, hay que trasladar e intentar buscar soluciones a las preocupaciones ciudadanas». Todo ello en momentos de crisis económica, que coincide con el asentamiento de la sociología (Urrutia, 2012), tanto de la sociología académica como de la profesional.

\subsection{La creación de la titulación de Sociología en la Facultad de Ciencias Sociales y de la Información de la Universidad del País Vasco (UPV/EHU)}

En esta fase de consolidación de la sociología en el País Vasco, a la Facultad de Sociología y Ciencias Políticas de la Universidad de Deusto, se añade la creación de la Facultad de Ciencias de la Información de la Universidad del País Vasco (UPV/EHU).

En el curso 1977-1978 había comenzado a funcionar la Unidad de Ciencias de la Información de Leioa, centro dependiente de la Facultad de Periodismo de la Universidad Autónoma de Barcelona, que «impartía sus clases en locales cedidos por la Universidad de Bilbao, debiéndose su creación inicial a una voluntad casi autogestionaria de los alumnos vascos que estudiaban Periodismo en la capital catalana» (De Pablo y Rubio, 2006: 268). Siendo todavía Unidad delegada, se crea una comisión formada por Pérez-Agote, González Portilla y García Herrera, profesores de Sociología, Historia Contemporánea y Derecho Constitucional, respectivamente, que básicamente funcionaba como equipo decanal y se dedicaba a gestionar la Unidad delegada (Maestro, 2013). Esta comisión será la encargada de gestionar también el tránsito a la Facultad de Ciencias de la Información ${ }^{6}$. 
González Portilla (2013) recuerda así ese periodo:

«Lo que sí configuramos es un grupo de presión interna, por áreas de conocimiento, para hacer valer un poco los intereses generales de lo que era la Unidad. Y entonces yo estaba por Historia, Alfonso Pérez-Agote por Sociología, Miguel Ángel García Herrera por Derecho, y así alguna gente más».

Y más adelante, continúa el autor:

«La constitución de la Facultad de Ciencias de la Información se hace a partir de un grupo de presión de gente que estábamos allí dentro. [...] Pero había dificultades administrativas importantes porque en ese momento estaba reformándose la Universidad, primero con el gobierno de Suárez, que no consigue aprobar la ley [la LAU]. [...] Teníamos el apoyo de los rectores [de la Universidad del País Vasco y de la Autónoma de Barcelona] para hacer la facultad, pero no había el sistema institucional que lo hiciese viable. [...] y fue con el final del gobierno de Suárez, cuando empiezan a moverse las cosas, y es cuando al final se consigue el apoyo para crear la Facultad de Ciencias de la Información. Porque se desbloquea ese impasse político que afectaba gravemente a toda la universidad española. Pero este proyecto ya venía desde el año 79. Se tenía el respaldo de las dos universidades para crear la facultad» (González Portilla, 2013).

Con la fundación de la Universidad del País Vasco en 1980, la Unidad de Ciencias de la información adquiere plena autonomía y se constituye en Facultad de Ciencias de la Información en 1981 (De Pablo y Rubio, 2006), siendo decano comisario ${ }^{7}$ Celestino del Arenal, catedrático de Relaciones Internacionales de la Universidad Complutense de Madrid (Maestro, 2013). Aunque en sus inicios la facultad solo ofertaba la licenciatura en Ciencias de la Información, pronto atraerá a docentes de los diversos ámbitos de las ciencias sociales y jurídicas, entre ellos la sociología.

De esta manera, en lo que respecta a la docencia de materias de sociología, van incorporándose como profesores de la facultad en este periodo: Miranda, Minondo, Pérez-Agote, procedentes del Departamento de Sociología de la Facultad de Ciencias Económicas y Empresariales de Sarriko; Gurrutxaga, Llera, Calvo, Mardones, Urrutia, Mata, que habían sido docentes de la Facultad de Ciencias Políticas y Sociología de la Universidad de Deusto; Muñoz, Unzeta, Aierdi, Larrinaga. El primer director de este departamento ha sido PérezAgote, quien lo dirige entre 1978 y 1985 (Urrutia, 2013b).

de Ciencias de la Información en los primeros planes de estudio, se debió a que el origen de la propia facultad estaba asentado en estas tres disciplinas (Maestro, 2013).

7 Decano comisario era, en ese periodo de transición política, un título que se adquiría sin pasar por los procedimientos habituales, es decir, que la elección de este cargo académico no se llevaba a cabo a través de la Junta de Facultad, sino que era nombrado directamente por el rector, en este caso por el rector Martín Mateo (González Portilla, 2013). 
En 1985, tras la aprobación de la Ley Orgánica 11/1983, de 25 de agosto, de Reforma Universitaria, con el primer gobierno de Felipe González, es elegido director del Departamento de Sociología, E. López-Aranguren, que había obtenido en 1984 la cátedra de Sociología ${ }^{8}$ en la Facultad de Ciencias Económicas y Empresariales de la UPV/EHU (Urrutia, 2013b).

En 1987, concretamente con fecha de 30 de junio de 1987, siguiendo la normativa del Real Decreto 2360/1984, de 12 de diciembre, sobre departamentos universitarios, y "de acuerdo con la notificación del Decanato de la Facultad de Ciencias de la Información y con la aprobación previa del Rectorado de la Universidad del País Vasco /EHU» (Departamento de Sociología, 1987), se constituye oficialmente el Departamento de Sociología de la Universidad del País Vasco (UPV/EHU). Posteriormente, es elegido director del Departamento de Sociología en el primer Consejo de Departamento, tras su constitución como departamento universitario, Pérez-Agote — quien ocupa de nuevo este cargo académico hasta 1991-y secretario, Aierdi. (Departamento de Sociología, 1987; Urrutia, 2013b).

Por otro lado, de forma paralela al desarrollo de la titulación de Ciencias de la Información (Especialidades: Periodismo y Publicidad) ${ }^{9}$, para cuya impartición había sido creada la facultad, se planteó la creación de una sección específica para las ciencias sociales y, en concreto, la sociología y la ciencia política.

Es en 1987, con el equipo decanal de González Portilla ${ }^{10}$ (1987-1990), equipo de consenso formado por Díaz Mancisidor —ya fallecido—, del área de Periodismo; Zallo, de Comunicación; Gurrutxaga, de Sociología; López Basaguren, de Derecho, y el propio González Portilla, de Historia, que se plantea la posibilidad de traer los estudios de sociología y ciencias políticas a la Facultad de Ciencias de la Información de la UPV/EHU, dado el capital humano, compuesto de profesores e investigadores cualificados, con que ya contaba la facultad en esas áreas (González-Portilla, 2013). Sin embargo, la tarea no resultará fácil, dada la oposición, en un primer momento, del equipo rectoral de Barberá, por la presión ejercida desde la Universidad de Deusto (González Portilla, 2013), que ya ofertaba esos estudios. Pero a pesar de las presiones, finalmente, en 1988 la facultad comenzará a ofertar la licenciatura en Ciencias Políticas y Sociología ${ }^{11}$ (Especialidades: Sociología Industrial-Urbana,

\footnotetext{
${ }^{8}$ Como se ha podido apreciar a lo largo de estas páginas, la primera cátedra de Sociología de la Facultad de Económicas de Bilbao la obtiene Jiménez Blanco en 1962, quien la ocupa hasta el año 1964, en que se traslada a Málaga. Hasta el año 1971, en que la obtiene Moya, se produce por tanto un vacío, que se repite entre la marcha de este último, que en 1974 se desplaza a Madrid para dirigir el Instituto de Ciencias de la Educación de la UNED y poner en marcha su Departamento de Ciencias Sociales (Ramos, 2013), y la cátedra de E. López-Aranguren de 1984. Este último también se trasladará a Madrid en 1990, para ocupar la cátedra de Sociología en la Universidad Carlos III de Madrid (Urrutia, 2013b). Según González Portilla (2013), estos vacíos encuentran su explicación en el hecho de que la Universidad de Bilbao, después Universidad del País Vasco (UPV/EHU), es una universidad periférica y los catedráticos que vienen de fuera del País Vasco tienden a marcharse, sobre todo a Madrid.

${ }_{9}^{\text {Cfr. BOE n. }}{ }^{\circ}$ 10, de 12 de enero de 1983.

${ }^{10}$ González Portilla ha sido el primer decano de la Facultad de Ciencias de la Información de la UPV/EHU, elegido democráticamente en Junta de Facultad. Durante su decanato (1987-1990) se gestionará el tránsito a la Facultad de Ciencias Sociales y de la Información de la UPV/EHU.

${ }^{11}$ La Universidad del País Vasco ha sido la cuarta universidad pública en España en ofertar la titulación de Ciencias Políticas y Sociología, tras la Universidad de Madrid (1973), la de Barcelona (1986) y la UNED (1987) (Navarrete, 1990).
} 
Sociología Política y Ciencias Políticas) ${ }^{12}$, en lo que supondría su posterior transformación primero en Facultad de Ciencias Sociales y de la Información, y después en la actual Facultad de Ciencias Sociales y de la Comunicación.

Así, se van incorporando también como profesorado del Departamento de Sociología en la Facultad de Ciencias Sociales y de la Información: Arpal, procedente de la Facultad de Filosofía y Ciencias de la Educación de la UPV/EHU; Arregui, Domínguez —fallecido en 1998—, Fernández Sobrado, Garmendia, Homobono, Ispizua, Iturrioz, Manzanos, Martínez de Luna, Oleaga, Rivas, Rodríguez, Tejerina, Uriarte y Urrutia Izaguirre (Departamento de Sociología, 1990a, 1990b). Y son también profesores del Departamento de Sociología, en otros centros: E. López-Aranguren, Larrea, Vicario, Barrenetxea, Martínez Monje y Bazo, en la Facultad de Económicas y Empresariales de Sarriko; Espí, en la Escuela de Empresariales de Donosti; Mateos, Usategui, Larrañaga, Maneros, en la Escuela de Magisterio de Bilbao (Departamento de Sociología, 1989, 1990a; Moreno, 1990).

\subsection{El boom de la demanda de estudios sociológicos: el descubrimiento de la Sociología por la administración pública y el desarrollo de la sociología profesional}

Según Gurrutxaga (1989: 10), el «proceso de crecimiento de la Sociología ha dependido, en gran medida, de su «descubrimiento» por la Administración autónoma y municipal, así como por un sector de empresarios privados. La estructura académica es significativa, en tanto en cuanto reproduce la profesión, pero en el crecimiento de la Sociología parece jugar un papel más significativo las oportunidades laborales». Coincide en este sentido con Urrutia (2012), cuando este último dice:

«En aquellos primeros años, los años 1980, entre lo que demanda la Administración pública (ayuntamientos, diputaciones y Gobierno vasco) más lo que demandan [...] [los] primeros grupos de investigación en estudios de mercado, van acogiendo a los licenciados en sociología que entonces existían».

En lo que se refiere a las experiencias de una sociología más profesional, continúan en esta década algunos de los grupos o gabinetes de investigación, que ya se habían creado a finales de los años 1970, y se crean otros nuevos. Urrutia (2012, 2013a) destaca los siguientes:

1. «Grupos de investigación social aplicada: el ya mencionado AZTERKA (1978/1984); DATLAN (1985/1991), formado por ex-alumnos de sociología de la Universidad de Deusto y economistas; y SIADECO (Sociedad de Investigación Aplicada para el Desarrollo Comunitario), todavía activa, y que en los años setenta, y fundamentalmente en Gipuzkoa, desarrollaba investigaciones sociolingüísticas y socioeconómicas.

${ }^{12}$ Cfr. BOE n. ${ }^{\circ} 261$, de 31 de octubre de 1989 y BOE n. ${ }^{\circ}$ 297, de 12 de diciembre de 1989. 
2. Gabinetes de estudios sociológicos, marketing, socio-economía: el gabinete de sociología CINDES S. L., dirigido por Ruiz Olabuenaga; IKEI-Instituto Vasco de Estudios de Investigación (Kutxa-Donostia); ARALDI, que proviene de la economía aplicada, e IKERTALDE, que son grupos formados por economistas. Son empresas que tienen su origen en esta época y siguen subsistiendo en la actualidad. Aprovechan el desarrollo que está viviendo la administración pública y realizan estudios de carácter estadístico en demanda de información socio-demográfica, por ejemplo, y van contratando a algunos sociólogos. IKERFEL, que todavía se mantiene, fundado por Fernández de Luco, que fue profesor de la Facultad de Ciencias Políticas y Sociología de la Universidad de Deusto. Se dedica a los estudios de mercado y atrae a algunos sociólogos como profesionales; METRA SEIS.

3. Gabinetes de análisis de recursos humanos, organización: Organización y Desarrollo (1982/1984), dirigido por Uriarte, profesor de sociología industrial de la Facultad de Sociología de la Universidad de Deusto, en el que participaron sociólogos que habían estudiado la rama de sociología industrial. Es un grupo que intervino en muchas investigaciones de análisis de la organización empresarial, pero también de la administración pública. Se puede decir que de este grupo surgieron las consultorías de carácter empresarial».

Es esta sociología profesional la que va ser criticada por algunos sociólogos vascos, mostrando nuevamente las tensiones entre diferentes concepciones de la sociología, en este caso entre sociología académica y sociología profesional, al hablar de la sociología practicada en la Facultad de Ciencias Políticas y Sociología de la Universidad de Deusto, y que nos recuerdan las ya observadas en el caso de la sociología española, con autores como Perpiñá (1967) y Lamo de Espinosa (1992) ${ }^{13}$. Según Gurrutxaga (1989: 10-11):

«[...] la estrategia profesionalista de Deusto sobredeterminaba las diversas concepciones de la Sociología. [...] La estrategia de Deusto tiene una clara vocación comercial. Deusto no pretende formar intelectuales, sino expertos, oferentes para un imaginario mercado, todavía por crear y definir en su mayoría, que desconocen no sólo al sociólogo como trabajador, sino, por supuesto, a la Sociología como ciencia. [...] El perfil del sociólogo de Deusto tiene una clara orientación hacia salidas técnicas o profesionalistas. Se trataría de un sujeto capaz de enfrentarse a las demandas de la Administración, de gabinetes de investigación de mercados, de empresas, etc. [...] Desde esta perspectiva, no se puede afirmar que Deusto haya construido una Sociología para el País Vasco, sino todo lo más podemos sostener la hipótesis de que ha preparado profesionales, conocedores de un conjunto de técnicas que les permiten acceder al mercado de trabajo profesional».

Y que también reflejan las tensiones entre la sociología teórica y la sociología empírica. Así, continúa el autor:

13 Sobre este particular, véase Martin Aranaga (2016). 
«[En Deusto] se valora más el conocimiento de las «últimas» técnicas de investigación que las polémicas teoréticas. [...] Creo que esta orientación profesionalista y técnica de la Facultad de Deusto tiene diversas consecuencias para la evolución de la Sociología. La primera es la imposibilidad de crear, desde estas premisas, un cuerpo sólido para comprender la complejidad de la sociedad vasca contemporánea. En segundo lugar la tecnocratización de la Sociología y la peligrosa identificación de sociólogos-técnicos sociales. En tercer lugar la creación de una conciencia de que la Sociología sólo puede ser empírica» (Gurrutxaga, 1989: 11).

\subsection{La Asociación Vasca de Sociología: del compromiso a la profesionalización}

Como se ha mencionado en el apartado anterior, la puesta en marcha de la nueva administración democrática (autonómica, foral y local) ha constituido una de las oportunidades laborales más atrayentes para la sociología. Para Aierdi (2007: 229), esta «circunstancia, además de facilitar la creación de «salidas profesionales», estimuló también la promoción de cursos complementarios de formación y la aparición de las primeras «investigaciones aplicadas», en línea con los surveys de la tradición anglosajona».

Así, la Asociación Vasca de Sociología (AVS/ESE) se orienta, desde su compromiso socio-político inicial, hacia la profesionalización (Aierdi, 2007; Urrutia, 2013b) y, en su primer boletín, Gizartea, publicado en diciembre de 1983, con el nombre «Asociación Vasca de Sociólogos», la Junta Directiva hace referencia explícita a la «necesaria profesionalización de los sociólogos como grupo» (Aierdi, 2007). No obstante, es este compromiso socio-político inicial, que se encuentra en el origen de la fundación de la Asociación Vasca de Sociología, el que ha propiciado que la AVS/ESE haya optado por mantener su estatuto asociativo y no tanto por reconvertirse en colegio profesional, aunque haya habido momentos y personas dentro de la Asociación que sí se lo han planteado (Zubero, 2009).

Entre 1983 y 1985, la Asociación presenta un ritmo de actividad inusitado. Durante este periodo, se organizan las Jornadas de Sociología de la Salud (1983) y las Jornadas de Planificación Social de la Administración Local; también, desde la Asociación, se está indirectamente presente en la organización del I Congreso de Sociolingüística (1984) y en el I Congreso de Sociología de la Vida Cotidiana (Aierdi, 2007). En este tiempo, preside la «Asociación Vasca de Sociólogos» Ruiz Olabuenaga ${ }^{14}$.

Entre 1986 y 1990, bajo las presidencias de Urrutia y Llera, se promueve la revista Inguruak (Ibídem, 2007), la organización del III Congreso Español de Sociología, celebrado en

\footnotetext{
${ }^{14}$ Llera ha sido el primer Presidente de la Asociación Vasca de Sociología, Ruiz Olabuenaga el segundo, y Urrutia el tercero (Asociación Vasca de Sociólogos, 1986, 1988; Urrutia, 2013b). Desde su fundación, ha existido el consenso de turnar la presidencia de la AVS/ESE entre el profesorado de la Universidad de Deusto y el de la Universidad del País Vasco (Urrutia, 2012, 2013b). Así, han sido también Presidentes/as de esta Asociación (Aierdi, 2007): María Luisa Setién (U. de Deusto), Aierdi (UPV/EHU), María Silvestre (U. de Deusto), Amaia Alonso, procedente del mundo extra-académico. Actualmente, preside la hoy Asociación Vasca de Sociología y Ciencia Política (AVSP), Zubero, profesor titular de Sociología de la UPV/EHU.
} 
San Sebastián en 1989 (Llera, 1989; Navarrete, 1990; Urrutia, 2012), el desarrollo de los cursos especializados de inserción laboral para licenciados en Sociología y se estrechan los lazos de colaboración con la Federación de Asociaciones de Sociología del Estado Español (FASEE) (Aierdi, 2007).

Para Urrutia (2012), el III Congreso Español de Sociología, celebrado en San Sebastián en 1989, bajo el lema "La Sociología frente a los retos del siglo xxi», y en cuya organización participa activamente la Asociación Vasca de Sociología, marca, de alguna manera, un «cambio de perspectiva de lo que era la sociología entendida como unos estudios de compromiso socio-político a una sociología vista también como unos estudios con opciones profesionales».

\section{LA SOCIOLOGÍA VASCA DESDE 1990: CRECIMIENTO Y CRISIS}

\subsection{El crecimiento de la Sociología: la institucionalización definitiva de la sociología en el País Vasco}

Entre los años 1990-2000, se produce el mayor crecimiento de la sociología en la Universidad del País Vasco (UPV/EHU), tanto en términos de plazas ofertadas de profesorado en el Departamento de Sociología como del número de personas que se licencian en esta carrera. Así lo indican las palabras de Gurrutxaga (2013):

[...] en el curso 1988-89 desde la Facultad de Ciencias de la Información, que es como se llamaba entonces, se crean las Licenciaturas de Sociología y Ciencia Política. [...] Esto es muy importante porque en cuestión de muy poco tiempo, cuatro años escasos, se crean casi 70 plazas nuevas para profesores de Ciencia Política y Sociología. Ahí se da una gran emergencia de personas, de trabajos, de líneas de investigación. [...] De tal manera que en gran parte la sociología, lo que diríamos el gran ciclo de la sociología se acaba cerrando a comienzos de la década de los años 90.

Y los datos que se exponen en la Tabla 1.

Sin embargo, este periodo en el que culmina la institucionalización de la sociología en el País Vasco, no va a estar exento de tensiones. Tensiones debidas a desencuentros personales, causas políticas y, también, a discrepancias en cuanto a la concepción de la sociología que se quiere primar desde la academia. Nosotros centraremos nuestra atención en estas últimas.

En 1991, se crea el Departamento de Estudios Internacionales y Ciencia Política —más tarde Departamento de Ciencia Política y de la Administración- de la UPV/EHU, siendo su primer director Llera, hasta entonces profesor del Departamento de Sociología. Ello se 
Tabla 1. Licenciados/as en Sociología UPV/EHU. 1993 a $2013^{15}$

\begin{tabular}{|c|c|}
\hline Promoción & $\begin{array}{c}\text { N. }{ }^{\circ} \text { de personas } \\
\text { egresadas }\end{array}$ \\
\hline 1993 & 59 \\
\hline 1994 & 56 \\
\hline 1995 & 67 \\
\hline 1996 & 62 \\
\hline 1997 & 120 \\
\hline 1998 & 263 \\
\hline 1999 & 223 \\
\hline 2000 & 190 \\
\hline 2001 & 112 \\
\hline 2002 & 60 \\
\hline 2003 & 52 \\
\hline 2004 & 55 \\
\hline 2005 & 67 \\
\hline 2006 & 51 \\
\hline 2007 & 35 \\
\hline 2008 & 34 \\
\hline 2009 & 43 \\
\hline 2010 & 35 \\
\hline 2011 & 30 \\
\hline 2012 & 29 \\
\hline 2013 & 37 \\
\hline
\end{tabular}

Fuente: Elaboración propia a partir de los datos extraídos de Setién (1998), Lanbide (2007, 2012) y Secretaría de la Facultad de CC. Sociales y de la Comunicación de la UPV/EHU (2013).

produce tras la estancia de este último en la Universidad de Yale, donde se ha formado con Linz, y donde ha adquirido una concepción de la ciencia política más en el sentido anglosajón, es decir más empírica, caracterizada por los estudios electorales y el análisis de los partidos políticos, de las políticas públicas (politics) y de los sistemas representativos (Urrutia, 2013b). Posteriormente, Llera se vincula a un pequeño grupo de catedráticos que han estudiado en Estados Unidos, y que se oponen a la corriente tradicional de la ciencia política ligada al derecho. Así, comienzan a crearse los Departamentos de Ciencias Políticas en las universidades de Madrid, Barcelona y el País Vasco, que funcionan como un

${ }^{15}$ Los estudios de la Licenciatura en Ciencias Políticas y Sociología comienzan en la UPV/EHU en 1988-89, con dos especialidades Sociología y Ciencia Política. La primera promoción de Licenciados data del curso 1992-93. La primera modificación del plan de estudios de esta carrera, el denominado "plan nuevo", de 4 años, se llevará a cabo en 1995-96, licenciándose su primera promoción en 1998-99, y dando lugar a dos titulaciones distintas: Licenciatura en Sociología y Licenciatura en Ciencia Política y de la Administración. 
lobby (Urrutia, 2013b). La cristalización de este lobby será la fundación de la Asociación Española de Ciencia Política y de la Administración (AECPA), con el objetivo de abrir el camino a la ciencia política como área de conocimiento y como grupo profesional.

A este nuevo departamento se suman también Mata y Uriarte, procedentes del Departamento de Sociología y, procedentes de otros departamentos de la Facultad de Ciencias Sociales y de la Información de la UPV/EHU, Aldecoa, Barcena, Zubiaga, Letamendia, Ibarra, entre otros docentes.

En 1992, siendo director del Departamento de Sociología el profesor Urrutia, se produce la división departamental. La consecuencia de ello es la creación de un nuevo departamento, el Departamento de Sociología II, cuyo primer director será Pérez-Agote, y al que se suman, entre otros profesores, Gurrutxaga, Arpal, Tejerina, Unceta, Arregui y Fernández Sobrado. Urrutia continuará como director del Departamento de Sociología originario, ahora Departamento de Sociología I, hasta 1994 (Urrutia, 2013b).

Entre las razones que se esgrimen para explicar esta división, algunas muestran nuevamente las tensiones ya observadas entre diferentes concepciones o cosmovisiones de la sociología. Así, para Pérez-Agote (2013), las razones tienen que ver con el interés por una sociología con un fuerte componente teórico y empírico a la vez.

«El departamento se hizo muy grande y hay un momento en que pensamos en dividirlo. [...] ¿Razones?, pues yo creo sensibilidad, igual yo era muy sensible a lo académico y a la investigación [...]. La función que me atribuí siempre fue el desarrollo de la investigación con un componente teórico muy fuerte y un componente empírico muy fuerte, las dos cosas. En parte para procurar, como había mucha gente que provenía de Deusto, que era una sociología fundamentalmente aplicada y poco teórica, pues para poder compensar ese origen. Unos que venían de Deusto, otros que veníamos de ninguna parte, de Paris, pero muy tardíamente, pues hacer una cosa muy teórica y muy empírica a la vez» (Pérez-Agote, 2013).

Según Urrutia (2012, 2013b), las razones que explican la división departamental deben ser matizadas, y aunque reconoce el interés de algunos profesores por una sociología más aplicada, se trataba sobre todo de hacer atractivos los estudios de sociología para los estudiantes y de orientar la carrera fundamentalmente a la profesión. Dice Urrutia (2012):

«Había en el Departamento de Sociología de la UPV/EHU una cultura latente que nos partía. [...] Yo creo que sí hay una coincidencia entre un grupo de profesores que se orienta más hacia la sociología en términos de abstracción, que se concreta en los debates de carácter muy teórico respecto a los problemas identitarios, y otro bloque de profesores que se orientan más hacia la investigación empírica, hacia la aplicación, hacia la sociología aplicada, hacia los problemas sociales más apremiantes». 
Y, más adelante, el autor matiza:

«Nosotros éramos gente más pegados a lo que son, y aquí viene un problema serio, a lo que son las salidas profesionales de los alumnos. [...] Estábamos muy preocupados por las salidas profesionales porque ya estaban las promociones, entonces entendíamos que había que orientar la carrera fundamentalmente a la profesión. [...] Las razones... nosotros estábamos aquí preocupados por la sociología urbana, los métodos de investigación, es decir, cómo hacer viable que la carrera tuviese un interés para la gente que empezaba a estudiar sociología. Esa es la razón» (Urrutia, 2012).

De alguna manera, se reproduce en el interior del Departamento de Sociología el mismo rechazo a la profesionalización de la sociología, que ya se advirtió en la crítica a la sociología practicada en la Universidad de Deusto, realizada por Gurrutxaga en un artículo publicado en «Cuadernos de Alzate» en 1989. Así lo indican las palabras de Urrutia (2013b):

«Y luego internamente aquí se volvió a reproducir la tensión [entre quienes tenían una concepción de la sociología más teórica y quienes teníamos una concepción más aplicada], porque estábamos una serie de gente que decíamos «no podemos estar aquí en la abstracción», o no solamente. [...] Esa tensión la hemos tenido siempre, porque lo de AZTERKA, todo lo que ha seguido, es justamente cómo lo que nosotros hemos estudiado, lo convertimos en una herramienta que sirva para que la gente pueda desarrollar su profesión. Eso sí ha sido constante. Y eso tiene que ver con la Asociación [Vasca de Sociología], tiene que ver efectivamente con esa diferencia [entre una concepción más teórica o más aplicada de la sociología] y también lo que genera aquí el haberse producido la ruptura del departamento. Y luego hemos perdido el norte».

En 1994, la Escuela de Trabajo Social de Vitoria-Gasteiz, centro dependiente de la Iglesia y apoyado financieramente por la Caja Vital, se integra plenamente en la Universidad del País Vasco (De Pablo y Rubio, 2006). Así, el profesorado del área de conocimiento de Trabajo Social y Servicios Sociales pasa a formar parte del Departamento de Sociología I, lo que conllevará un incremento importante del número de profesores de este departamento, y supondrá posteriormente un cambio en su denominación, ya que actualmente recibe el nombre de Departamento de Sociología y Trabajo Social, al integrar dos áreas de conocimiento: Sociología, por un lado, y Trabajo Social y Servicios Sociales, por otro.

Tanto el Departamento de Sociología y Trabajo Social como el Departamento de Sociología 2 tienen su sede en la Facultad de Ciencias Sociales y de la Comunicación de la UPV/ EHU. El primero está compuesto por 50 docentes e investigadores y 4 investigadores predoctorales, agrupados en torno a las dos áreas de conocimiento mencionadas más arriba, Sociología y Trabajo Social y Servicios Sociales ${ }^{16}$. Su funcionamiento se extiende a los tres

${ }^{16}$ La relación de profesoras y profesores de los Departamentos de Sociología y Trabajo Social y del Departamento de Sociología 2 de la UPV/EHU puede verse en Universidad del País Vasco (2016c, 2016d). 
campus de la Universidad del País Vasco (UPV/EHU), y tiene docencia en doce grados, tales como el grado de Sociología, en la Facultad de Ciencias Sociales y de la Comunicación (campus de Bizkaia); el grado de Trabajo Social, en la Facultad de Relaciones Laborales y Trabajo Social (campus de Álava); o los grados en Educación Primaria y Educación Infantil, impartidos en los tres campus de la UPV/EHU (Universidad del País Vasco, 2016a). El Departamento de Sociología y Trabajo Social se ha responsabilizado en consecutivos bienios de una serie de Programas de Doctorado, que han tenido como base el análisis de las nuevas tendencias sociales en diferentes ámbitos de la sociedad contemporánea. En la actualidad, es corresponsable, junto al Departamento de Antropología y Filosofía de los valores, del Máster Oficial «Estudios Feministas y de Género» (Ibídem, 2016a).

El Departamento de Sociología 2 cuenta con 25 profesores/as, 3 investigadores/as adscritos a proyectos de investigación, 14 investigadores/as en formación, una investigadora postdoctoral, un investigador Ikerbasque, y una secretaria administrativa (Universidad del País Vasco, 2016b). Este Departamento presta docencia en once grados repartidos en los tres campus de la UPV/EHU. Si bien su mayor capacidad docente se desarrolla en la Facultad de Ciencias Sociales y de la Comunicación, el Departamento de Sociología 2 se encuentra presente en otras facultades y centros, como Relaciones Laborales y Trabajo Social, Farmacia, Aulas de la Experiencia, etc. Además de ofrecer docencia de grado, el Departamento de Sociología 2 es responsable del doctorado «Modelos y Áreas de Investigación en Ciencias Sociales», del Máster Oficial del mismo nombre y del Título Propio «Hizkuntza Plangintza» (Postgrado Especialista de Universidad) (Ibídem, 2016b).

\section{2. ¿Crisis de la Sociología y/o crisis de la titulación de Sociología? La consecuencia de una profesionalización inconclusa}

Todos los sociólogos vascos entrevistados coinciden en señalar que es en la década de los años 2000 cuando se inicia la crisis de la Sociología en el País Vasco, o en todo caso de la titulación específica de Sociología, ya que la materia de sociología se imparte en otras titulaciones. Así lo reflejan los datos de la Tabla 1, expuestos más arriba, sobre el número de licenciados/as en Sociología en la UPV/EHU en el periodo 2002-2013. Y el hecho de que la titulación de Sociología ya no se oferte en la Universidad de Deusto, debido a la falta de demanda de estos estudios en esta universidad (Ruiz Olabuenaga, 2013). No hay que olvidar además que esta última titulación constituye la titulación matriz de la disciplina sociológica en el País Vasco. Ni tampoco que se trata de una crisis que no es exclusiva de nuestro territorio, como se puede observar en el caso de la sociología española (Alvira, 2001; Pérez Yruela, 2007), y de la que comienzan a advertirse ciertos indicios en el contexto francés (Piriou, 2008).

Según Ruiz Olabuenaga (2013), las razones de esta crisis se deben al propio éxito de la sociología en el País Vasco, que ha llevado a ofertar esta carrera en varias universidades, públicas y privadas. Y en este sentido coincide con Urrutia (2012), cuando también menciona el exceso de oferta de estudios de sociología en nuestra Comunidad. 
«Eso ocurre por la competencia de la universidad pública. La desaparición de la Facultad de Sociología en la Universidad de Deusto tiene que ver con la insostenibilidad de la sociología tal y como estaba entonces. Era insostenible que hubiera dos facultades para la demanda que había» (Urrutia, 2012).

Urrutia (2012) también hace referencia al desajuste entre los alumnos que estudian la carrera de sociología y la demanda real de estos titulados por parte del mercado de trabajo.

"Hay una ruptura entre la gente que accede y la demanda de ciencia social que hay. [...] Hay una inflación que mata el propio desarrollo equilibrado de la profesión. Porque sí había demanda de sociólogos, pero no en la medida de los alumnos que acudían a las aulas. [...] Y yo creo que entra un poco en crisis no solo en Euskadi, sino también en España, pero que se deriva de la afluencia importante que tienen todas las carreras, y fundamentalmente las carreras de ciencias sociales. Y ahí se hace un poco el crack y luego viene la caída actual» (Urrutia, 2012).

Y como todo ello guarda relación con cómo se han configurado las universidades y las carreras en España.

«Esto tiene que ver también con una inflación en los servicios que toda administración autonómica cree desarrollar en ese espíritu de consolidación del estado autonómico. Cada comunidad autónoma y cada ciudad creen que debe establecerse una universidad y además una universidad generalista. Es un modelo muy mecanicista del Estado, de un Estado que se descentraliza, y que entienden que esa descentralización cada una de sus partes deben reproducir de una manera mimética unos servicios, en este caso universitarios. [...] Hay un crecimiento espectacular de las universidades públicas con una demanda también muy fuerte de profesorado que en la siguiente década hace crisis. Ese crecimiento de universidades, de carreras y de profesores, a su vez con la implantación de los nuevos planes de estudio, mantiene un espíritu tribal en el que no hay interdisciplinariedad. Cada uno defiende su territorio [...]. Esa reproducción metastásica de los estudios, de la universidad, es la que acaba entrando en crisis, que es lo que tenemos ahora» (Urrutia, 2012).

Para Pérez-Agote (2013), la crisis de la sociología tiene que ver con el hecho de que el referente empírico sobre el que los sociólogos han construido la sociedad, ya no existe.

«La sociología está en crisis en todo el mundo por una razón fundamental, que es porque la sociología ha sido una ciencia de la sociedad, y la sociedad tal como los sociólogos la han construido, la han construido con un referente empírico obvio y evidente que eran las sociedades estatales nacionales, y eso ya no existe» (Pérez-Agote, 2013).

Ruiz Olabuenaga (2013) hace referencia igualmente a "una sociedad que no existe», a los cambios operados en la sociedad y a la necesidad, por tanto, de cambiar la ciencia 
que estudia la sociedad, es decir la sociología. De ahí la necesidad de una «nueva sociología».

«[...] si lo que ha cambiado no es la sociología, lo que ha cambiado es la sociedad, y la sociedad ésta es tan nueva y tan distinta que o cambia la ciencia que estudia la sociedad, o si no, no se entiende nada. [...] Estamos todavía usando unos conceptos de una sociedad que no existe. Totalmente falsa. [...] Hace falta una nueva sociología porque la sociedad es nueva» (Ruiz Olabuenaga, 2013).

Según este autor, no se ha sabido «aceptar que la sociedad cambia y que hay que cambiar la sociología», no se ha sabido "entrar en el conocimiento de estos cambios profundos que ahora necesitan hacer los sociólogos».

Por su parte, Gurrutxaga (2013) disecciona de manera crítica los problemas y desafíos a los que se enfrenta la sociología en el momento actual: 1) la hegemonía de las ciencias experimentales y la investigación tecnológica, frente al papel prácticamente inexistente, según este autor, de la investigación social; 2) el no haber conseguido que los estudios de sociología se conviertan en un oficio; 3) la incapacidad de los sociólogos para relacionarse con la sociedad. Según sus palabras:

«Por una parte, tenemos que enfrentarnos con una hegemonía que en estos momentos tienen las ciencias experimentales y toda la investigación tecnológica, donde la investigación social no juega prácticamente ningún papel. Es decir, nos hemos quedado en el furgón de cola de los programas de investigación, de los repartos de fondos, etc. Esto es un hecho, el mundo ha girado, ha cambiado, y las humanidades y las ciencias sociales en general copamos un lugar claramente periférico o secundario, cuando no estamos en la periferia de la periferia, que puede ocurrir también en algunas situaciones. Y esto es un dato difícil de asumir, sobre todo por las consecuencias que tiene, pero imposible de no plantearse.

En segundo lugar, yo creo que tenemos otro gran problema y es que no hemos conseguido que los estudios de sociología se conviertan en un oficio. Ha habido carreras que lo han conseguido, psicología, por ejemplo, hay un oficio claro. [...] Pero si la impugnación sobre la sociología no está fuera de la sociología. La impugnación sobre la sociología la llevan a cabo los propios sociólogos. Es decir, en este momento fuera, en la calle, en las empresas, etc., lo que son muchos de los conocimientos que nosotros somos capaces de crear, de jugar con ellos, están siendo muy demandados. Nuestro gran problema es por qué no ofrecemos o no somos capaces de acercarnos a todo ese mundo y ofertarlos. Nuestro fracaso tiene que ver con la institucionalización de los propios estudios de sociología, nuestro fracaso está en la propia Universidad y en nuestras incapacidades.

Hay un tercer gran tema, no hemos sabido tampoco relacionarnos con la sociedad. Esto es muy curioso porque una ciencia, cuyo objeto básico es dar ese conocimiento... resulta que no ha sido capaz de buscar su lugar bajo el sol, ni de encontrar 
ligámenes. Existen por supuesto ligámenes y estrategias personales, pero no como profesión» (Gurrutxaga, 2013).

Para Leonardo (2012), el «drama de la sociología» no solo en el País Vasco, sino también en España, es la falta de "perfil profesional» del sociólogo, y ello se debe al hecho de que nunca se ha planteado en los planes de estudio el tipo de saber que necesita la persona que se va a dedicar al mundo profesional de la sociología. Se observa, por tanto, una cierta coincidencia entre este autor con el segundo problema apuntado por Gurrutxaga, si bien este último hace referencia a la falta de «oficio» de sociólogo, y Leonardo incide más en la inexistencia de una «profesión» de sociólogo. Así, dice Leonardo (2012):

"Quizás soy demasiado drástico en mis pensamientos, pero parto de la hipótesis de que precisamente es ese el drama de la sociología en España, que no ha habido un perfil profesional. [...] En España, [...] el drama de la sociología, y hoy estamos pagando ese drama, es que se ha confundido la sociología como disciplina con las necesidades de la sociología desde la universidad. Dicho de otra forma, se ha enseñado, se ha preparado, se ha habilitado a los estudiantes que se han sentado en las aulas para que repitan los roles del sociólogo que está en la universidad, desde la perspectiva de los propios profesores. Esa es mi tesis central. Es decir, jamás se ha planteado en los planes de estudio, en el enfoque, en la disciplina, por distintas razones muy complejas, la idea de qué habilidades, qué instrumentos, qué tipo de saber necesita una persona que se va a dedicar al mundo profesional de la sociología. Los planes de estudio están pensados para desarrollar un sociólogo, a poder ser crítico con el sistema, porque si no, no entras en los parámetros de comme il faut. [...]. Entonces, ¿qué ha pasado? Las temáticas, los problemas, el contenido del saber que se ha inculcado y las problemáticas a las que se han dedicado históricamente los sociólogos, insisto podemos poner la excepción de lo que es el mundo de la investigación de mercados, pues han sido los problemas de la academia, hasta el punto, y hay muchos indicadores pero el más visible, cuando uno organiza un Congreso de Sociología en España, el 99'9\% de las intervenciones son o bien de profesores de la universidad o de gente que está haciendo el doctorado. Del mundo profesional no hay nadie. Como si ese mundo no existiese. Desde mi punto de vista se ha difuminado el rol profesional del sociólogo. ¿Eso significa que no hay sociólogos que trabajen? No, hay sociólogos y sociólogas que trabajan en la medida en que han demostrado habilidades o que demuestran competencias en determinadas materias» (Leonardo, 2012).

Esta carencia de perfil profesional repercute además en la imagen que la sociedad tiene del sociólogo.

"[L]a sociedad no ha sido capaz de percibir qué es lo que hace un sociólogo. Y el ejemplo más notable es la universidad. El ejemplo más notable es que los padres y las madres que envían a sus hijos a la universidad dicen: ¿y eso para qué sirve? Cosa que no se les ocurre hacer por ejemplo con un trabajador social. Todo el mundo sabe que un trabajador social da ayuda, está en la administración, tiene un perfil profe- 
sional. ¿Qué ha pasado? Que unas cosas sobreviven porque tienen una utilidad social y otras no» (Leonardo, 2012).

Este último aspecto también es resaltado por Pérez-Agote (2013), para quien existe un problema de imagen de la sociología como profesión.

«[En] España no hay imagen de la profesión. Se cree que todos los sociólogos son investigadores, por lo tanto la sociedad piensa que somos inútiles, que no valemos más que para hacer cosas que no valen para nada, y cuando alguien quiere hacer algo práctico no se le ocurre llamar a un sociólogo, se le ocurre yo creo llamar antes a un politólogo, que saben sacar determinadas cosas más concretas y piensan que los sociólogos no. Y creo que es porque los sociólogos, y ahí sí tenemos culpa los sociólogos, no hemos difundido una imagen profesional que sea correcta, que esté bien hecha (Pérez-Agote, 2013).

Y Ruiz Olabuenaga, en un artículo publicado en 1998 en la revista Inguruak, titulado «20 años de sociología vasca: retos y riesgos de una profesionalización incompleta», y que recoge la conferencia inaugural del IV Congreso Vasco de Sociología, reflexiona sobre la Sociología en el País Vasco, haciendo especial énfasis en su aún incompleta profesionalización. Dice este autor:

«La Sociología como profesión reclama unos estándares de excelencia académica, de rigor metodológico, de deontología personal, de solidaridad corporativa, de seguridad ocupacional, de disponibilidad de recursos, etc. que, hoy por hoy, distan mucho de haber sido alcanzados por muchos de nosotros. No diré que sigamos siendo francotiradores, pero sí que nuestra profesionalización sigue siendo inadmisiblemente imperfecta» (Ruiz Olabuenaga, 1998: 8).

Por último, interesa resaltar aquí la comparación que realizan los autores entre la Sociología y otras disciplinas del campo de las Ciencias Sociales, tales como la Psicología (Gurrutxaga, 2013), el Trabajo Social (Leonardo, 2012) o la Ciencia Política (Pérez-Agote, 2013), cuyo modelo profesional adoptado les ha permitido configurar profesiones - psicólogo, trabajador social, politólogo-, con mayor visibilidad y reconocimiento social, según los autores, que la Sociología. También, que la identificación excesiva del sociólogo a la investigación (Pérez-Agote, 2013), parece limitar la imagen que la sociedad tiene de este profesional.

\section{CONCLUSIONES}

En este artículo se han tratado de plasmar, en primer lugar, los hechos más significativos del proceso de institucionalización y profesionalización de la Sociología en el País Vasco. De esta manera, se han identificado cuatro momentos o fases de este proceso. 
La primera de ellas abarca el periodo 1953-1973, y en ella destacan como hechos relevantes: el Instituto de Estudios Sociales de la Universidad de Deusto y la cátedra de Sociología de la Facultad de Ciencias Políticas, Económicas y Comerciales de la Universidad de Bilbao. La segunda fase, de 1974 a 1980, comprende la creación de la Facultad de Ciencias Políticas y Sociología en la Universidad de Deusto y la fundación de la Asociación Vasca de Sociología (AVS/ESE), así como la aparición de las primeras experiencias de una sociología aplicada de corte más profesional. La tercera es la fase de consolidación de la sociología en el País Vasco, años 1980-1990, con la creación de la titulación de sociología en la Facultad de Ciencias de la Información -más tarde Facultad de Ciencias Sociales y de la Información y en la actualidad Facultad de Ciencias Sociales y de la Comunicación- de la Universidad del País Vasco (UPV/EHU), y el boom de la demanda de estudios sociológicos, debido al descubrimiento de la sociología por parte de la administración pública vasca y al desarrollo de la sociología profesional. Por último, a partir de los años 90 se produce un crecimiento de la sociología, tanto en términos de plazas ofertadas de profesorado en esa disciplina en la universidad pública vasca, como de titulados en sociología. Ello hasta comienzos de la década de los años 2000, en que comienza a advertirse, según los interlocutores clave entrevistados, una cierta crisis de la sociología en el País Vasco, o en todo caso de la titulación específica de Sociología. Es lo que indican los datos recogidos para la elaboración de este artículo, y el hecho de que la titulación de Sociología ya no se oferte en la Universidad de Deusto, titulación, esta última, que constituye la titulación matriz de la disciplina sociológica en el País Vasco.

En cuanto a las razones de esta crisis, los sociólogos entrevistados refieren las siguientes: éxito de la Sociología en el País Vasco, que ha llevado a ofertar esta carrera en varias universidades; desajuste entre oferta y demanda de estudios de Sociología; la configuración de las universidades y carreras en España; que el referente empírico sobre el que los sociólogos han construido la sociedad ya no existe; hegemonía de las ciencias experimentales y la investigación tecnológica, frente al papel prácticamente inexistente de la investigación social; no haber conseguido que los estudios de sociología se conviertan en un oficio; la dificultad de los sociólogos para relacionarse con la sociedad; falta de "perfil profesional»; problema de imagen de la sociología como profesión; incompleta profesionalización de la sociología.

Así, ya se trate de falta de «oficio» de la sociología (Gurrutxaga, 2013), de inexistencia de «perfil profesional» (Leonardo, 2012), de problema de «imagen profesional» de la sociología (Leonardo, 2012; Pérez-Agote, 2013) o de "profesionalización incompleta» (Ruiz Olabuenaga, 1998), lo cierto es que los autores parecen coincidir en lo inacabado del proceso de profesionalización de la sociología, también en el País Vasco, y en que la responsabilidad de esta situación corresponde, en definitiva, a los sociólogos y a la academia (Leonardo, 2012; Gurrutxaga, 2013; Pérez-Agote, 2013).

En segundo lugar, se buscaba verificar la hipótesis planteada al inicio de este artículo que consistía en comprobar, en el caso de la sociología vasca, la existencia de tensiones entre diferentes cosmovisiones o concepciones de la sociología que han atravesado todo el reco- 
rrido de la sociología vasca. Nos referíamos a las tensiones u oposiciones entre la sociología como disciplina científica y la sociología como disciplina aplicada, entre la sociología teórica y la sociología empírica, la sociología académica y la sociología profesional, entre otras. Pensamos que las páginas que nos preceden han mostrado la existencia de estas tensiones que, a nuestro entender, pueden haber provocado que el proceso de profesionalización de la Sociología en el País Vasco resulte inacabo.

En conclusión, se podría decir que los dos objetivos planteados al inicio de este artículo, parecen haberse alcanzado, aunque no plenamente. Se ha logrado el objetivo consistente en comprobar la hipótesis de partida mencionada más arriba y, en este sentido, se ha demostrado la existencia de tensiones entre diferentes concepciones o cosmovisiones de la sociología a lo largo del recorrido de la sociología vasca. Pero no tanto el referido al relato de lo acontecido en la sociología vasca. Por un lado, es evidente que las personas entrevistadas han sido y siguen siendo figuras representativas de la sociología en el País Vasco, pero también es cierto que no están todas las que son o fueron. La imposibilidad de entrevistarlas a todas -cuestión a la que se enfrenta en algún momento todo investigador- se encuentra en el origen de estas ausencias. Por otro lado, reconocemos que en nuestro trabajo han quedado sin tratar algunas cuestiones clave. Es el caso del papel jugado tanto por los grupos de investigación como por los congresos vascos de sociología en el proceso de institucionalización de la sociología vasca. Por las razones expuestas consideramos que nuestro trabajo constituye una aproximación al objeto de estudio, la institucionalización y profesionalización de la Sociología en el País Vasco, que debería tener una continuación en futuras investigaciones que abordaran aquellos temas que aún no han sido analizados.

\section{REFERENCIAS BIBLIOGRÁFICAS}

Aierdi, X. (2007). Perfil de la Asociación Vasca de Sociología y Ciencia Política. Revista Española de Sociología, n. ${ }^{\circ}$, pp. 227-233.

Alvira, F. (2001). Presente y futuro de la sociología en España. Revista Española de Sociología, n. ${ }^{0}$ 1, pp. 49-59.

Asociación vasca de sociólogos (1986). Directorio. INGURUAK-Revista de Sociología, n. ${ }^{\text {0 }}$ 0, p. 3.

Asociación vasca de sociólogos (1988). Directorio. INGURUAK-Revista de Sociología, marzo 1988, p. 11.

Beltrán, M. (2009). In Memóriam: José Jiménez Blanco, catedrático de Sociología. El País. Disponible en: http://elpais.com/diario/2009/01/14/necrologicas/1231887601_850215.html

Del Campo, S. (2001). Historia de la sociología española. Barcelona: Ariel. 
De Pablo, S. y Rubio, C. (2006). Historia de la UPV/EHU (1980-2005). Bilbao: Universidad del País Vasco.

Departamento de sociología (1987). Acta de constitución del Departamento de Sociología de la UPV/EHU y Acta de Consejo de Departamento, 30/06/1987. Leioa: Universidad del País Vasco (UPV/EHU).

Departamento de sociología (1989). Acta de Reunión del Departamento de Sociología, 24/11/1989. Leioa: Universidad del País Vasco (UPV/EHU).

Departamento de Sociología (1990a). Acta de Consejo de Departamento, 28/09/1990. Leioa: Universidad del País Vasco.

Departamento de Sociología (1990b). Acta de Reunión del Departamento de Sociología, 16/11/1990. Leioa: Universidad del País Vasco.

Gurrutxaga, A. (1989). Sociología vasca: la necesidad de su descubrimiento. Cuadernos de Alzate-Revista vasca de la cultura y de las ideas, n. ${ }^{\circ} 12$, pp. 5-17.

Iturrate, J. L. (1975). Apéndice: Sociología en España. Notas para su historia. En J. Cazeneuve y D. Victoroff (eds.), La Sociología (pp. 548-618). Bilbao: Mensajero.

Lamo de Espinosa, E. (1992). La sociología española desde 1939. En J. Ibáñez (dir.), Sociología (pp. 119-130). Madrid: Universidad Complutense de Madrid.

Lanbide (2007). Sociología. Promoción 2003 -UPV/EHU, LANBIDE- Observatorio Mercado de Trabajo. Disponible en: http://www.lanbide.net/plsql/Pu_BuscarPublicacion (20 de mayo de 2009).

Lanbide (2012). Sociología. Promoción 2008 —UPV/EHU, LANBIDE- Observatorio del Mercado de Trabajo. Disponible en: http://www.lanbide.net/plsql/Pu_BuscarPublicacion (8 de mayo de 2013).

Llera, F. J. (1989). La sociología española frente a los propios retos. Cuadernos de AlzateRevista vasca de la cultura y de las ideas, n. ${ }^{0} 12$, pp. 19-24.

Martin Aranaga, I. (2016). La inserción profesional de las sociólogas y sociólogos vascos: formación, empleo, identidad, profesión. Tesis Doctoral. Leioa: Universidad del País Vasco (UPV/EHU).

Moreno, L. (1990). Sociología en la España finisecular. En S. Giner y L. Moreno (Comps.), Sociología en España (pp. 71-103). Madrid: CSIC.

Navarrete, L. (1990). La organización académica de la sociología en España». En S. Giner y L. Moreno (Comps.), Sociología en España (pp. 275-278). Madrid: CSIC. 
Pérez Yruela, M. (2007). El retorno de la Sociología. Revista Española de Sociología, n. ${ }^{\circ}$ 7, pp. 13-26.

Perpiña, A. (1967). Nueva y vieja sociología. Discurso de recepción del Académico de número. Madrid: Real Academia de Ciencias Morales y Políticas.

Piriou, O. (2008). Que deviennent les diplômés de sociologie? Un état de la discipline et de son avenir, Socio-logos. Revue de l'association française de sociologie, $\mathrm{n} .^{\circ} 3$. Disponible en: http://socio-logos.revues.org/1622 (13 de agosto de 2012).

Ramos, R. (2013). Semblanza de D. Carlos Moya Valgañón. Premio Nacional de Sociología y Ciencia Política 2010, CIS. Disponible en: http://www.cis.es/cis/ opencms/ES/6_formacion/PremioNacionalSociologia/Semblanzas/CarlosMoya. html?semblanza=true (14 de octubre de 2013).

Ruiz Olabuenaga, J.I. (1998). 20 años de Sociología vasca: retos y riesgos de una profesionalización incompleta. Inguruak - Revista Vasca de Sociología y Ciencia Política, n. ${ }^{0} 20$, pp. 7-24.

Setién, M. ${ }^{2}$ L. (1998). 20 años de Sociología en el País Vasco. 1977-78 a 1996-97. Titulados y Profesionales. Ponencia presentada en el IV Congreso Vasco de Sociología, Bilbao.

Universidad de Deusto (1997). Reseña histórica, Facultad de Ciencias Políticas y Sociología de la Universidad de Deusto. Disponible en: http://www.deusto.es/cas...uc01/fac06c/ fac0603c.htm (11 de diciembre de 1997).

Universidad del País Vasco (UPV/EHU) (2013). Información institucional. Antecedentes históricos. Disponible en: http://www.ehu.es/p200-content/es/contenidos/informacion/ historia_upvehu/es_histori3/historia_3.html (14 de octubre de 2013).

Universidad del País Vasco (UPV/EHU) (2016a). Departamento de Sociología y Trabajo Social. Presentación. Disponible en: http://www.ehu.eus/es/web/sociologiaytrabajo social/aurkezpena (25 de marzo de 2016).

Universidad del País Vasco (UPV/EHU) (2016b). Departamento de Sociología 2. Presentación. Disponible en: http://www.ehu.eus/es/web/sociologia2/aurkezpena (25 de marzo de 2016).

Universidad del País Vasco (UPV/EHU) (2016c). Departamento de Sociología y Trabajo Social. Personal del departamento. Disponible en: http://www.ehu.eus/es/web/ sociologiaytrabajosocial/langileak (17 de junio de 2016).

Universidad del País Vasco (UPV/EHU) (2016d). Departamento de Sociología 2. Presentación. Personal del departamento. Disponible en: http://www.ehu.eus/es/web/ sociologia2/nortzuk-gara (17 de junio de 2016). 